\title{
Hybrid systems of gold and silver nanoparticles generated on cellulose surfaces
}

\author{
A.P. Carapeto*, A.M. Ferraria*, P.A. Carvalho**, P. Brogueira**, S. Boufi*** and A.M. Botelho do Rego* \\ *Centro de Química-Física Molecular and IN, Instituto Superior Técnico, University of Lisbon, Av. Rovisco \\ Pais, 1049-001 Lisbon, Portugal \\ **ICEMS, Instituto Superior Técnico, University of Lisbon, Av. Rovisco Pais, 1049-001 Lisbon, Portugal \\ ***Laboratoire des Sciences des Matériaux et Environnement, Uni. of Sfax, BP 802-3018 Sfax, Tunisia
}

Developing methods for immobilizing metallic nanoparticles (NP) onto different surfaces is a subject of fundamental interest, as nanometric structures enable the appearance of novel properties, absent in bulk or even in micrometric dimensions. In fact, the presence of NP with different size and shape on surfaces can induce substantial modifications on their electronic, optical, magnetic, physical or chemical proprieties. This will be very useful for many applications in different areas ranging from electronics to biomedicine $[1,2]$.

In this work, we use a simple, reproducible and economic method to grow metallic gold and silver NP directly on cellulose surfaces. A prior chemical activation of the alcohol groups on the cellulose surface is needed for grafting amino functions, which will complex silver and gold ions. Therefore, NP nucleation is only allowed and selectively accomplished on these seed coordination sites. The growth of NP is achieved by the interaction of aqueous dilute solutions of $\mathrm{AgNO}_{3}$ or $\mathrm{NaAuCl}_{4}$ with the cellulose surface. The procedure limits the generation of NP only to the cellulose surface, keeping the dispersion medium completely exempt of them. The resulting NP are, consequently chemically immobilized on the cellulose surface. The chemical anchoring of the NP to the surface avoids particle desorption and extends the lifetime of the resulting hybrid materials. Following this procedure, silver and gold NP could be successfully created on cellulose and this was supported by transmission electron microscopy (TEM), scanning electron microscopy (SEM) and atomic force microscopy (AFM).

The present AFM (Figure 1) study enabled to characterize the size and shape of silver NP: they are spherical and have a diameter of $\sim 15 \mathrm{~nm}$. SEM image of Ag NP (Figure 2) display the existence of NP agglomerates. Comparison of the two images suggests size and spatial distributions strongly non- uniform. It is now a challenge to deposit nanoparticles in an organized manner, achieving a regular surface functionalization of cellulose. In TEM (Figure 3) we see a sample where Ag and Au salts interacted simultaneously with the cellulose surface. Crossing the images with other information from analytical techniques, it seems that Ag NP and Au NP form mainly separately instead of presenting a core-shell structure. Also, there is evidence that larger $\mathrm{NP}$ are the ones made of silver which suggests that the final result is kinetically (and not thermodynamically) controlled.

References

1. Daniel M.C. et al., Chem. Rev., 104:293-346, 2004.

2. Ferraria A.M. et al., Langmuir, 26:1996-2001, 2010. 

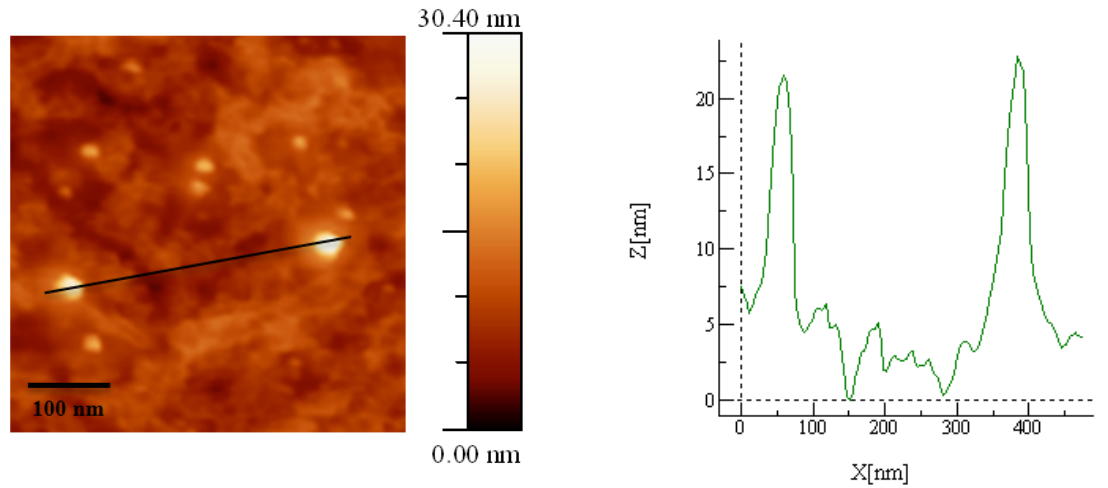

Figure 1. AFM image and profile of cellulose surface after Ag nanoparticles growth.

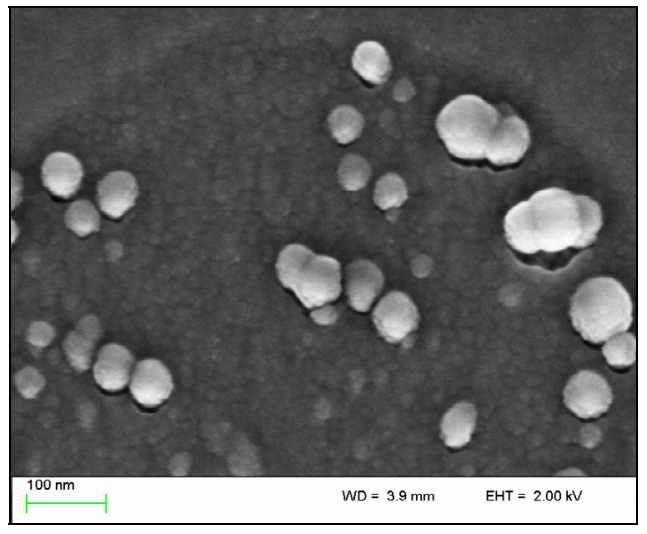

Figure 2. SEM image of cellulose surface after Ag nanoparticles growth.
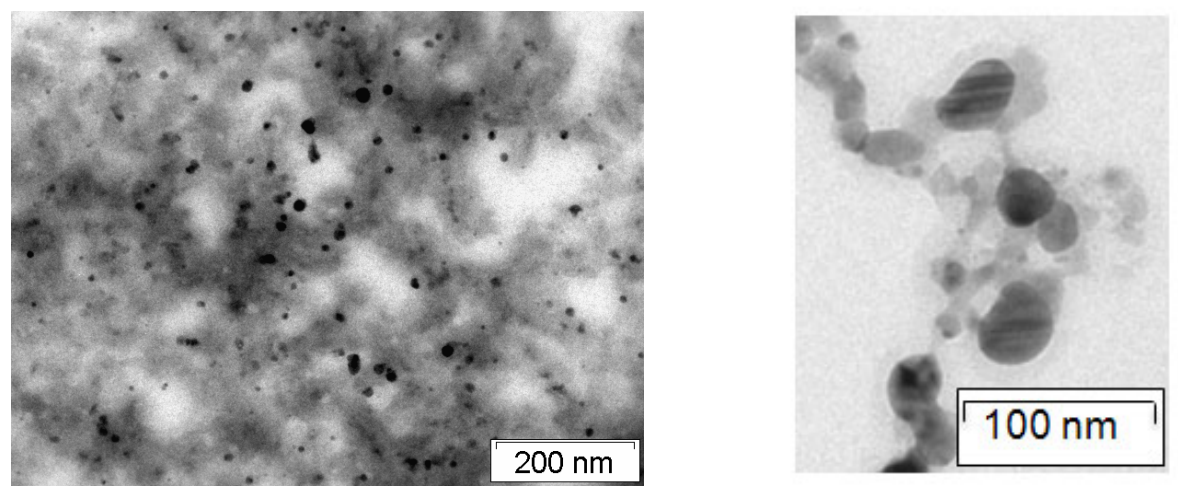

Figure 3. TEM images of cellulose surface after Ag and Au nanoparticles growth. In the detailed image we can see features usually assigned to defects typical of gold and silver.

The authors acknowledge FCT for A.M. Ferraria Grant SFRH/BPD/26239/2006, for A. P. Carapeto Grant SFRH/BD/75734/2011 and strategic projects PEst-OE/CTM/LA0024/2011 and PEst-OE/CTMUI0084/2011. 